\title{
CONCENTRACION DE RIQUEZA TERRITORIAL IMPONIBLE Y DE VARIAS CONTRIBUCIONES, POR PROVINCIAS, EN ESPAÑA, EN 1941
}

Por ser del máximo interés en orden al conocimiento de la vida económica de un país el grado de concentración de su riqueza y tributación, máxime si se discriminan las clases de riqueza y de tributos; y no existiendo-que nosotros sepamos-ningún estudio relativo a tal asunto en España, publicamos este primer principio de ensayo, utilizando los pocos datos que hemos logrado reunir, considerando como elemento individual de las series estadísticas la provincia española (y ello con la excepción de las que tienen contribución concertada en la mayoría de los tributos), lo cual reduce muchísimo el alcance práctico de nuestro trabajo en orden al conocimiento de la verdadera concentración de la riqueza española, para cuyo cálculo exacto se necesita que las series estadísticas contengan, en sus diversas rúbricas o epígrafes, el número de poseedores o de tributadores y la cuantía de lo que posee o tributa cada uno, a fin de clasificarlo globalmente por orden creciente desde el mínimo al máximo. $\mathrm{Si}$, al menos, conociéramos el número de contribuyentes en cada provincia, nuestro ensayo sería menos imperfecto, pero tampoco este dato hemos podido obtenerlo. 
Riqueza imponible y contribación (en

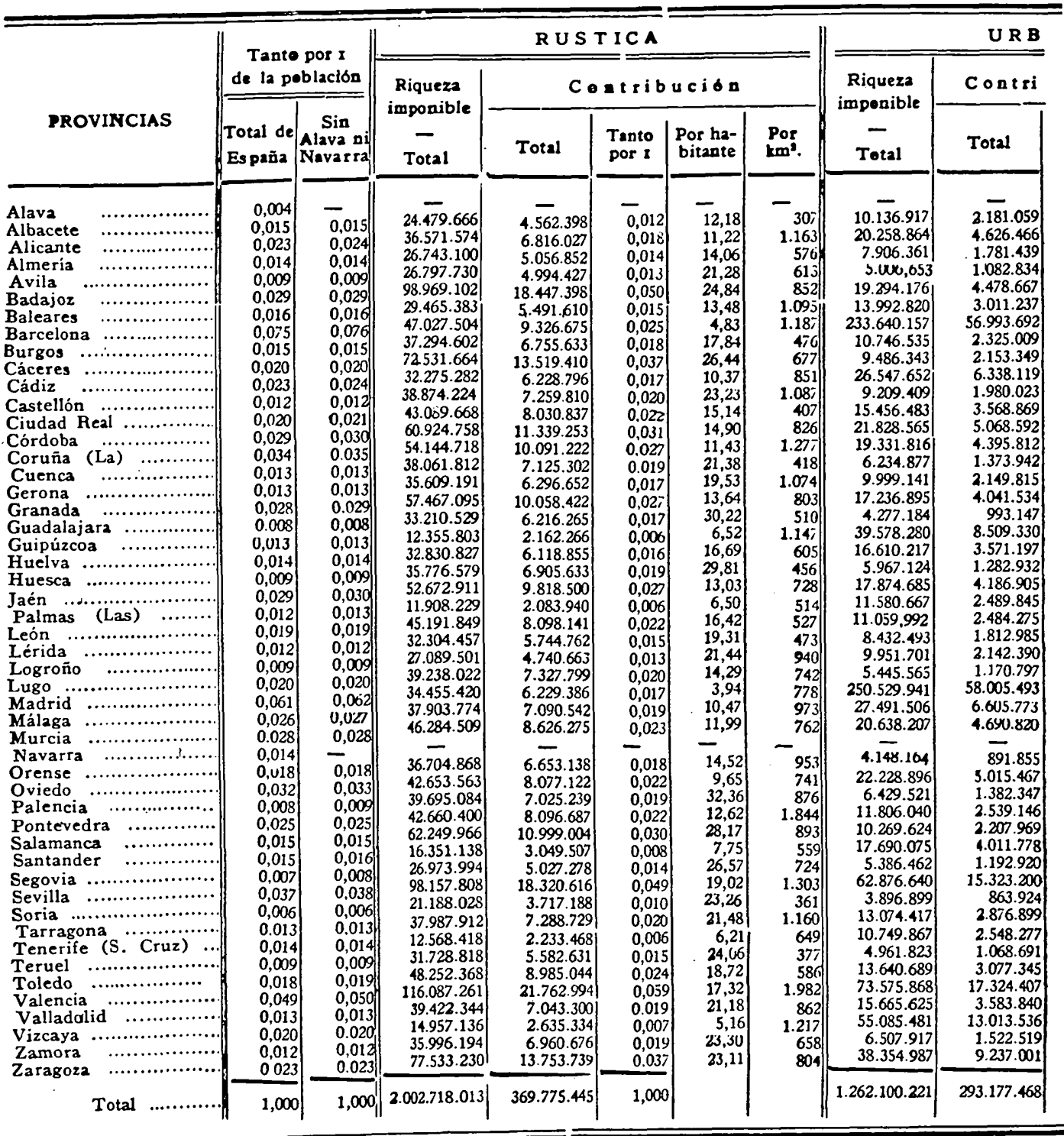


etas) por provincias. - Año 1941

\begin{tabular}{|c|c|c|c|c|c|c|c|c|c|c|c|c|c|}
\hline \multirow{2}{*}{$=$} & & \multicolumn{3}{|c|}{ INDUSTRIAL } & \multicolumn{3}{|c|}{ UTILIDADES } & \multicolumn{3}{|c|}{ PATENTES } & \multicolumn{3}{|c|}{$u^{\text {Total general de les cinco rubricas }}$} \\
\hline & & \multicolumn{3}{|c|}{ Contribucion } & \multicolumn{3}{|c|}{ Centribución } & \multicolumn{3}{|c|}{ Contribucion } & \multicolumn{3}{|c|}{ Contribución } \\
\hline $\begin{array}{r}\text { Ito } \\
1\end{array}$ & $\begin{array}{l}\text { Por ha- } \\
\text { bitante }\end{array}$ & Total & $\left|\begin{array}{l}T \text { aoto } \\
\text { por } 1\end{array}\right|$ & $\begin{array}{l}\text { Por } \\
\text { habi- } \\
\text { taste }\end{array}$ & Total & $\begin{array}{l}\text { T anto } \\
\text { por I }\end{array}$ & $\begin{array}{c}\text { Por } \\
\text { habi- } \\
\text { tante }\end{array}$ & Total & or & $\begin{array}{c}\text { Por } \\
\text { habi- } \\
\text { tante }\end{array}$ & Total & $\left|\begin{array}{c}T \text { anto } \\
\text { por } 1\end{array}\right|$ & $\begin{array}{l}\text { Por ha- } \\
\text { bitante }\end{array}$ \\
\hline & & - & - & - & 34.347 & 0.003 & $24,2=$ & - & - & - & & & \\
\hline $\begin{array}{l}007 \\
016\end{array}$ & 5,82 & 3.130 .613 & $0.00 ?$ & 8,36 & & 0,003 & 7,2 & $403.884^{\prime}$ & 0.000 & 1,08 & 12.998 .843 & 0.007 & 34. \\
\hline 116 & $7,6]$ & & 0.020 & 13.18 & & 0.009 & $23,2^{\mathrm{r}}$ & 1.416 .397 & 0.024 & 2,33 & & 0.014 & $\begin{array}{l}47,6 \\
32,3\end{array}$ \\
\hline & $4,9:$ & 2.20 & 0.006 & $\begin{array}{r}6,12 \\
6,97\end{array}$ & $\begin{array}{l}2.252 .942 \\
1.295 .739\end{array}$ & & 6.26 & & 0.006 & $\begin{array}{l}0,98 \\
1,09\end{array}$ & $\begin{array}{c}65 \\
13\end{array}$ & $0 . n 06$ & 39,47 \\
\hline 15 & $6,0.3$ & 5.16 & 0.01 .3 & $6.9^{-1}$ & & $\begin{array}{l}0.001 \\
0.004\end{array}$ & $\begin{array}{l}5,52 \\
5,26\end{array}$ & & 0. & 1,02 & $\begin{array}{r}9.263 .013 \\
32.757 .673\end{array}$ & 05 & 44,1 \\
\hline & 7.39 & 7.44 & 0,019 & 18,26 & 22 & & $\begin{aligned} 5.26 \\
18,86\end{aligned}$ & 1.035 .216 & 0,018 & & 24.670921 & $\begin{array}{l}0.016 \\
0.012\end{array}$ & 60,5 \\
\hline & 29.56 & 81.0 & 0.205 & $41, y 3$ & 183. & 0. & 94.8 & 10.0 & 0.169 & & 602 & $0.17 n$ & 176 \\
\hline ne & 6,14 & 960 & 0.011 & $11.3=$ & & & 11,3 & & & & U1.989 & 0.009 & 48 \\
\hline & 4,2 & & 0.009 & 6,86 & & & $5,1 \varepsilon$ & & 0. & & 155 & & \\
\hline 2 & $10.5 n$ & & 0.024 & 15.49 & & & $29.4:$ & 1.4 & & & & & \\
\hline & 6,54 & & $0.0 \mathrm{t}^{-}$ & $: 4,80$ & & & 10,72 & & 0. & & & & 57 \\
\hline & 6.7 & & 0.010 & $7.2 x$ & & & 6,98 & & & & & & \\
\hline & $6 \%$ & & 0,015 & 7,87 & & 0.007 & 7,86 & 1.2 & 0. & & & & \\
\hline & 4'98. & & 0.021 & $y, 62$ & & & 13,36 & & & & & & \\
\hline & 412 & 2 & 0. & $6,2 i$ & & 0. & 4.21 & & & & 99 & & \\
\hline & $6^{\prime} 6^{7}$ & & 0. & 7,66 & & & $13.6 \mathrm{n}$ & 1.4 & & & & & \\
\hline & $5^{\prime} 48$ & & 0 & 7,20 & & & 7.01 & & 0. & & & & \\
\hline & $4 ' 83$ & & & 7.3.3 & & & $10.1^{-1}$ & & & & & & \\
\hline$M x_{0}$ & $25^{\prime} 65$ & & & 33,25 & & & $114.2 r$ & 1.9 & 0. & 5.91 & 416 & & 85.51 \\
\hline & $9 ' 74$ & & & $y$ & & & 9.51 & & 0. & 1,30 & & & \\
\hline & $5 ' 54$ & & & 9 & & v, & 10.39 & & $0.00^{-}$ & & & & 57, \\
\hline & 5,56 & & 0, & 7 & & 0.005 & 5. & 89 & 0. & 1.9 & & 3 & 33 \\
\hline 0,009 & 77 & & 0,010 & 11, & & & $16.9 r$ & & 0. & 3,17 & & & 46 \\
\hline & 536 & & 0. & 8 & & & $11,3=$ & & & $1,2 \mathrm{U}$ & & & 42 \\
\hline 0,006 & $6^{\prime} 10$ & & 0.010 & 13.12 & & & 8.34 & & 0. & 2 & & & \\
\hline & 9,69 & & & 10,27 & & 0. & 20,4 & & 0. & $2,2,1$ & & & 70 \\
\hline & $2^{\prime} 2^{2}$ & & 0 , & 5,56 & & & 4.16 & & 0. & & & & \\
\hline & $36^{\prime} 7 ?$ & & 0. & 32,69 & 275 & & 714.33 & & 0. & 5.0 & 390 & & 252. \\
\hline 02 & 9275 & & 0.022 & 12,60 & & & 17,52 & & $u, t$ & 1.55 & & & \\
\hline 0,016 & 6.52 & $7,174,738$ & 0,018 & 9,97 & & & $10 . n^{\circ}$ & 1.24:489 & 0,020 & 1,68 & 28.956 .964 & & 40, \\
\hline & & & & & & 7 & 3,58 & - & - & - & & & \\
\hline & $1 '$ & & & 6,16 & & & 2,54 & & $0.0 n 9$ & 1,14 & & & \\
\hline & 5 & & & $11.4 \mathrm{~s}$ & & & 25,40 & & & $1,8^{-1}$ & & & \\
\hline 0,00 & $6^{\prime} 37$ & & 0 & 11,48 & & & 14. & & & $1.4^{-1}$ & & & \\
\hline w9 & 3.96 & & & & & & 14 & & 0 & $1,8 \mathrm{c}$ & & & 42, \\
\hline 0,008 & 5'65 & & 0,012 & 2,18 & & & & & $0 . n \ln !$ & $1,4-4$ & & & \\
\hline & $10^{\prime} 14$ & & 0.014 & 14,35 & & & 28.0 & & 0.01 & 1.8 & & 0 & \\
\hline & & & & 142 & & & 17. & & 0. & 2, & & & \\
\hline & $15.9 !$ & & & & & & 27. & 3.0 & 0. & & 74 & & 77,5 \\
\hline & 5,41 & & 0.0 & 8.67 & & & 6 & & 0. & 1 & & & 45 \\
\hline & 8. & & 0.016 & $18.0^{\circ}$ & & & 13 . & & 00 & $2,2 y$ & & & 63. \\
\hline & 7,0 & & & 78 & & & 12 & & & & & & \\
\hline & 4,6 & & 0. & 7,14 & & & 5.37 & & $0.00 \cdot$ & 0,82 & & & 41.99 \\
\hline & 6'41 & & & $75 \mathrm{~d}$ & & & 4,85 & & & $0.8 ?$ & & & 38.37 \\
\hline & 13,79 & $26 . i 4$ & 0.068 & 21,20 & 24.1 & 0.0 & 19.19 & 2.594955 & $0.04 \%$ & $2,0^{-}$ & 167 & & 73.6 \\
\hline & 0.78 & & 0.015 & 17,29 & & & 22.20 & & 0011 & $1,8^{-}$ & & & \\
\hline & 23,46 & 998 & 0.041 & 31.42 & $3 \omega .0 Y 0.3 / 0$ & 0.11 .3 & $197.0^{1}$ & 2.142459 & 0.036 & $4,1^{1}$ & 4870.3 & & 263 \\
\hline & 5.10 & 2.6 & & 3001 & & & 8 & & 0.004 & 0,82 & & & 47, \\
\hline & 15,52 & 11079631 & 0.028 & 18,6 ? & 21.160 .227 & 0.024 & 35,56 & 1.104 .116 & $0 \cap 3 n$ & 4,68 & 14 & & 95 \\
\hline 1,000 & & 27.68 & 1,000 & & .150 & & & 9.214 .193 & 1,000 & & $43.251^{\prime}$ & & \\
\hline
\end{tabular}


Cont ibación relativa ascendente por habitante en las provincias esp sñolas y su población relativa y acumulación de las mismas.-A ño 1941.

\begin{tabular}{|c|c|c|c|c|c|c|c|}
\hline \multirow{2}{*}{\multicolumn{2}{|c|}{$\begin{array}{c}\text { PROVINCIAJ̄ } \\
\text { (1) }\end{array}$}} & \multicolumn{2}{|c|}{$R$ ústlca } & & & \multicolumn{2}{|c|}{ Urbana } \\
\hline & & $\begin{array}{l}\text { ranto por } \\
1 \text { de la } \\
\text { población }\end{array}$ & $\begin{array}{c}\text { ranto por } \\
1 \text { de la } \\
\text { contribución }\end{array}$ & PROVINCIAS & & $\begin{array}{l}\text { Tanto por } \\
1 \text { de la } \\
\text { población }\end{array}$ & $\begin{array}{l}\text { Tanto por } \\
1 \text { de la } \\
\text { contribucion }\end{array}$ \\
\hline Madrid & $=$ & $0,062-$ & & Orense & $=$ & $0,018+$ & \\
\hline Barc & $=$ & $0,138+$ & $0,0.12 \div$ & Lugo & $=$ & $0,0.38+$ & $0,0 \mathrm{c}^{-1}$ \\
\hline Vizcaya & $=$ & $0,1=\bar{x}-$ & $0,049+$ & Pontevedra & $=$ & $0,0 \%, 3 \perp$ & 0,016 \\
\hline Tenerife $_{\text {(S. C.) }}$ ) & $=$ & 0,172 & $0,05.5 \div$ & Cuenca & $==$ & $0,07,5$ - & $0, y_{2}+$ \\
\hline Las Palmas & $=$ & $0,18_{5}-$ & $0,065+$ & Cáceres & $==$ & 0,015 - & $0,028 \dot{ }$ \\
\hline Guipúzcoa & $=$ & $0,128+$ & $0,057-$ & Teruel & $=$ & $0,10 \bar{j}+$ & $0,032+$ \\
\hline Santander & $=$ & $0,21 q t$ & $0.075+$ & Avila & $=$ & $0,114 \cdot t$ & $0.0 .35+$ \\
\hline Oviedo & $=$ & 0.247 & $0,09 ? \cdot-!$ & Guadalajara & $==$ & $0,1 \geq 2+$ & $0,10.39+$ \\
\hline Cádiz & $==$ & $0,271+$ & $0, \mathrm{I}_{4}+$ & Almería & $=$ & $0, i 35 \div$ & $0,0+j+$ \\
\hline Málaga & $=$ & $0,2 !, 8+$ & $0, I, j 3 r$ & Coruña & $=$ & $0,171+$ & 0,10501 \\
\hline A.lica & $=$ & 0,322 & $0.15 \mathrm{I}$ & Zamora & $==$ & $\mathrm{O}, \mathrm{IB}_{3}+$ & $0,0{ }^{5}+$ \\
\hline Cor & $=$ & $0,3 j 7+$ & $0,173 \div$ & León & $:=$ & $0,202 \div$ & $0,073+$ \\
\hline Miurcia & $==$ & $0,3^{x} j-$ & $0,201+$ & Soria & $=$ & $0,209+$ & $0,076 \div$ \\
\hline Alb & $=$ & 0,400 & $0,213 \div$ & Granada & $=$ & $0,237+$ & $0,0,0+$ \\
\hline Ponite & $=$ & $0,425+$ & $0,2.35+$ & Huesca & $=$ & $0,2-45+$ & $0,004+$ \\
\hline Jaé & $=$ & $0,455+$ & $0,252+$ & Taćn & $=$ & $0,270+$ & $0,1 \mathrm{cos}+$ \\
\hline $\mathrm{Bal}$ & $:=$ & 0,471 & $0,277+$ & $\mathrm{ca}$ & $=$ & $0,201+$ & 0,1 ii \\
\hline Gra & $=$ & $0,5(1)$ & $0,304+$ & Albacete & $=$ & $0,306+$ & $0,12,3 \cdot+$ \\
\hline & $==$ & $0,51+4$ & $0,3 ! 9+$ & & $=$ & $0.3 .39+$ & $0,1.11)+$ \\
\hline Lug & $=$ & $0,5.34+$ & $0,338+$ & $z z$ & $==$ & $0,310$. & $0,15+$ \\
\hline Orense & $=$ & $0,552+$ & $0,355+$ & Lérilda & $==$ & $0,3 \div 0+$ & $0,1 f, \mathrm{I}+$ \\
\hline Córc & $=$ & $0,3^{82}+$ & $0,387+$ & Burgos & $:=$ & $0,39 j+$ & $0,1(x) \div$ \\
\hline iudad Real & $=$ & 0,$6 ; 13 \div$ & $0,409+$ & Sego & $==$ & $0,40.3+$ & $7.3+$ \\
\hline Leó & $=$ & $0,0 \geq 2+$ & $0,436+$ & $\mathrm{Ca}$ & $=:$ & $0,4: 5+$ & $0,130+$ \\
\hline $\mathrm{Hu}$ & $=$ & זו ז- & $0,447+$ & ia & $=$ & $0, \therefore 24+$ & $185+$ \\
\hline Val & $==$ & $0,5^{8} 6+$ & $0,500+$ & To & $=$ & $0,44.3 \Gamma$ & $0,196+$ \\
\hline $\mathrm{Bu}$ & $=$ & $0,7 \because \cdot 5+$ & $0,524+$ & $\mathrm{Mu}$ & $=$ & 0,$1 ;:+$ & $0,212+$ \\
\hline Tol & $=$ & 0,7301 & $0.548+$ & ba & $=$ & $0,5: 1+$ & 0,$22 ;$ \\
\hline ev & $=-$ & $0,7,58+$ & $0,597+$ & $\mathrm{Ge}$ & $==$ & $0,514+$ & $0,2,36+$ \\
\hline Lé & $==$ & $0.7 \% 0+$ & $0,012+$ & Ciudad Real & $=$ & $0, \ddot{3} .35 \div$ & $0,2.18+$ \\
\hline e & $=$ & 0.783 & $0.62 \eta+$ & Tenerifs (S. C.) & $=$ & $0,54 y-\dot{r}$ & 0,$2 ; \%+$ \\
\hline dolid & $=$ & 0,7 wi + & $0,649+$ & Baleares & $:=$ & 0,$5 ;+$ & $0,267 \div$ \\
\hline Avi & $=-=$ & 0,805 & $0,60 x+$ & Alicante & $\because=$ & $0,539+$ & $0,283+$ \\
\hline Cue & $=$ & $0.818+t$ & $0,680+$ & Las $\mathrm{F}$ & $=$ & $0,0 \cup 2-$ & $0,2(1) 2 \therefore$ \\
\hline Log & $=$ & $0,8 \div 7+1$ & $0,093+$ & Tar & $==$ & $0,6 r_{j}+$ & $0,3: 2 \ldots$ \\
\hline Tar & $=$ & $0.8-10+$ & $0,713+$ & ño & $=$ & $0.122 .1+$ & $0,309+$ \\
\hline Zar & $=$ & $0,8 t: 3$ & $0,750+$ & $\mathrm{H}_{2}$ & $=$ & $0,6,38+$ & $0,321+$ \\
\hline Castellón & $==$ & $0.37 j$ & $0,770+$ & $M$ & $=$ & $0,6,65 \div$ & $0,3+4+$ \\
\hline & $=$ & $0.89_{1}+$ & $0.780+$ & nder & $=$ & $0.68 \mathrm{I}+$ & $0,35^{3} \cdot \dot{T}$ \\
\hline Z & $=$ & $0,89.3$ & $0,770+$ & Cá & $:=$ & $0,7 \mathrm{c} ;+$ & $0,380 \div$ \\
\hline Teru & $=$ & $0,912 \div$ & $0,8: 4+$ & Valladolid & $=$ & $0,713+$ & $0,392+\dot{T}$ \\
\hline $\mathrm{ad}$ & $==$ & 0,931 & $0,85.4+$ & & $=$ & 0,763 -上 & $0,4.5 i$ \\
\hline Các & $=$ & $0,951+$ & $0,901+$ & Zar & $=$ & $0,7),(-1$ & $0,4 S_{3} \div$ \\
\hline egovia & $=$ & $0,951)$ & $0,915+$ & Sevilla & $:=$ & $0,829+$ & $0,33$. \\
\hline inca & $==$ & $0,9 ?+t$ & $0,9.15+$ & & $=$ & $0,8+9+$ & $0,579+$ \\
\hline & $=$ & $0,5,33+$ & $0,964+$ & & $=$ & $0,86 \geq ;$ & $0.5054+$ \\
\hline Ldalajara & $:=$ & $0,9^{(1) !}$ & $0.00 \ddot{0}+$ & Barcelona & $=$ & $0,933+$ & $0, \operatorname{iin} 2+$ \\
\hline encia & $:=$ & $1, \infty 00$ & 1,000 & Iadrid & $=$ & $1,00 j$ & $I, x \times m$ \\
\hline
\end{tabular}

(1) Las provincias vienen sumadas unas a otras, acumulándose el total de todas las 704 anteriores; a la siguiente: Asi, el 2.0, será: Madrid + Barcelona; el 3:0, Madrid + Barcelona $704+$ vizcaya; el $4 .^{\circ}$, las anteriores, más Tenerife, etc: 
Contribación relativa ascendente por habitante en las provincias españolas y sa pobación relativa y acumulación de las mi mas. - Año 194 !.

\begin{tabular}{|c|c|c|c|c|c|c|c|}
\hline & & $\ln 1$ & $\mathbf{u} \leq \mathbf{t} \mathbf{r} \mathbf{t}$ a $\mathbf{l}$ & & & 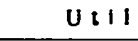 & lates \\
\hline $\begin{array}{l}\text { PROVINCIAS } \\
\text { (1) }\end{array}$ & & $\begin{array}{l}\text { Tanto I.or } \\
1 \text { de la } \\
\text { población }\end{array}$ & $\begin{array}{l}\text { Tanto por } \\
1 \text { te ia } \\
\text { contribucion }\end{array}$ & PROVINCIAS & & $\begin{array}{l}\text { ranto por } \\
1 \text { de la } \\
\text { poblución }\end{array}$ & $\begin{array}{l}\text { Tanto por } \\
1 \text { de la } \\
\text { contribui:ion }\end{array}$ \\
\hline Lugo & $=$ & $0, j 2 s,+$ & $0,0 n 7+$ & Orense & $=$ & $0,0 ! 8+$ & $0,0,2+$ \\
\hline Almeria & $=-$ & $0,0,3 t T$ & $0,013+$ & Navarra & $=$ & $0.932+$ & $0,1004+$ \\
\hline Orense & $=$ & $0.052+1$ & $0,020+$ & Lugo & $=$ & 0,$0 ; 2+$ & $0,006+$ \\
\hline Cuenca & $==$ & $0,36,5 \cdot 1$ & $0,025 \div$ & Cuenca & $=$ & $0, \because x_{5} \quad:-$ & $0,0: 28+$ \\
\hline Cáceres & $=$ & $0,035+$ & $0,03.4+$ & Toler!o & $=$ & $0,053+$ & $0,011+$ \\
\hline A vila & $=$ & $0,001+$ & $0,0,30 \div$ & Cáceres & $=$ & $0.10,3+$ & $0,017+$ \\
\hline Badajoz & $==$ & $0.13 .3 \mathrm{~T}$ & $0,0 j i+$ & Badajoz & $=$ & $0,1.32+$ & $0,0 i 9^{+}+$ \\
\hline Teruel & $=$ & $0.132 \ldots$ & $0,055+$ & Teruel & $==$ & $0,1, i \mathrm{I} \cdots$ & 0,010 t. \\
\hline Granada & $=$ & $0,1\} 1$ & $0,0,8,-$ & Avila & $=:$ & $0,:$ ii) $j-$ & $0,020+$ \\
\hline Ciudad Real & $=$ & $0,182+$ & $0,078 \cdot-$ & Jaén & $:=$ & $0,1 ? 0+$ & $0,2 \geq 5 \cdot t$ \\
\hline Guadalajara & $==$ & $0,1, y)+$ & $0,082+$ & Sor:a & $:=$ & $0.1 .35 t$ & $0,025+$ \\
\hline Tenerif $=$ (S. C.) & $=$ & 0,204 & $0,05+$ & Almería & $=-$ & $0,190+$ & $0,029+$ \\
\hline Tolerlo & $:=$ & $0.223 \cdot 1$ & $0,003+$ & Ciudad Real & $:=$ & $0,2 i r)+$ & $0,0.33+t$ \\
\hline Córdoba & $=$ & $0,2.3 .3 \cdot$ & $0,153+$ & Granada & $==$ & $0 .=47+$ & $0,0.39+$ \\
\hline Jaén & $=$ & $0,283-i$ & $0,128+$ & Albacete & $=$ & $0.252+$ & $0.042+$ \\
\hline León & $=$ & 0.302 i & $0,138+$ & Córdoba & $==$ & $0,20 i-i$ & $0.049+$ \\
\hline Albacete & $=$ & $0.3\{7 \div$ & $0,1.15+$ & Lérida & $\therefore=$ & $0,3 \div 3+$ & $0,052+$ \\
\hline Soria & $=$ & $0,3=3+$ & 0,149 - & Zamora & $:=$ & $0,315+$ & 0,$0 ; 5+$ \\
\hline Zamora & $=$ & $0,3.35$ & $0 . i 35 ;$ & $\mathrm{Hu}$ & $=$ & - & $0,0=7+$ \\
\hline Hu?lva & $=$ & $0,379 \div$ & $0.16_{5}+$ & $\mathrm{Mu}$ & $=$ & $0,3: 7+$ & $0,06,7+$ \\
\hline Coruña & $=$ & $0.38 .4+$ & $0,180 \div$ & Guadalajara & $\because=$ & $0,3^{h} ; i$ & $0,060+$ \\
\hline Ponteved:a & $=$ & $0,4: 49$ & $0,202+$ & Hue & $=$ & $0.374+$ & $0,072+$ \\
\hline Huesca & $:=$ & $0,4 B \cdot+$ & $0,2.2 \mathrm{~S}+$ & Castellón & $=:$ & $0.336+$ & $0,075+$ \\
\hline Murcia & $=$ & $0,44^{6}=$ & $0,2^{\prime} \div 5+$ & León & $=$ & $0.405 \div$ & $0.082+$ \\
\hline Burgos & $==$ & $0,4 \mathrm{I}$ & $0,237+$ & Burgos & $=$ & $0.420+$ & $0,087+$ \\
\hline Segovia & $=$ & 0,459 -1 & $0.242+$ & Tenerif = (S. C.) & $=$ & $0.734+$ & $0.06 .2+$ \\
\hline Oviedo & $=$ & $0.532 \div$ & $0,2045+$ & Salamanca & $\therefore=$ & $0.4 i n+$ & $0.007+$ \\
\hline Palencia & $=$ & $0,511+$ & $0,272 \div$ & Tar & $=:$ & $0,4^{(i 2}+$ & $0,102+$ \\
\hline Las Palmas & $:=$ & $0,5: 1+$ & $0,282+$ & Ali & $\therefore=$ & $0.435+$ & $0,111+$ \\
\hline Sevill & $=$ & $0.512+$ & $0.312+$ & Cor & $=$ & $0.51)+$ & $0.52: 1+$ \\
\hline anca & $=$ & $0,3,7 \div$ & $0.3 \geq 4$ & Ger & $:=$ & $0.532+$ & $0,129+$ \\
\hline Málaga & $:=$ & $0,604+$ & $0.3 .15{ }^{\circ}+$ & edra & $=$ & $0.557+$ & $0,130 \div$ \\
\hline Lér & $-=$ & $0,6: 0 \dot{0}$ & $0.355+$ & Pale & $=$ & $0.565+$ & $0,1.13+$ \\
\hline Alicante & $=$ & 0,679 & $0,: 70+$ & Las Paln & $=$ & $0.575+$ & $0.149+$ \\
\hline nider & $=$ & $0.6,=5 \quad i$ & $0.3 \% 0+$ & Segovia & $=$ & $0.58_{4} \cdot r$ & $0.1 ; .3+$ \\
\hline llón & $=$ & $0,0 n 8 \div$ & $0,4 \cap 2+$ & Má & $=$ & $0.510+$ & $0,165+$ \\
\hline Cádiz & $=-$ & $0 . \ln 3 ?+$ & $0.426+$ & Bale & $=$ & 0.026 & $0,1-5+$ \\
\hline $\log$ & $:=$ & $0.70 t+$ & $0.4 \therefore ; 1-$ & $\mathrm{Va}$ & $=-$ & $0.175 \div$ & 0,202 fo \\
\hline olid & $=$ & $0,714+$ & $0.4 i_{1}+$ & & $=$ & $0 . t) 94+$ & $0.207 t$ \\
\hline Gerona & $:=$ & $0.7 .27 r$ & $0,4^{\text {n. }} \cdot 1 \cdot+$ & Valladolid & $==$ & $0.67 \%$ & $0,215+$ \\
\hline T^rragonz & $==$ & $0,740 \perp$ & $0.480+1$ & Ala & $=$ & $0.7171-$ & $0.218+$ \\
\hline Raleares & $=:$ & $0,7: 1,+$ & $0.499+$ & & $=$ & $0, \ddot{733+}$ & $0.242+$ \\
\hline Zaragoza & $==$ & $0.7 ;: 1$ & $0.527+$ & Sevilla & $=$ & $0,700+$ & $0.272+$ \\
\hline cia & $=z$ & $0, \therefore, 11)+$ & $0.51) 5+$ & Santander & $=$ & $0.7^{2} 5 \ldots$ & $0,2 S_{1}+$ \\
\hline Vi & $:=$ & $0.8 .19+$ & $0.63^{2}+$ & Cádız & $=$ & $0.8 \cap S+$ & $0,304+$ \\
\hline Madrd & $=$ & $0.010+$ & $0 .-67+$ & Zaragoza & $\therefore=$ & $0,8,1+$ & $0.328+$ \\
\hline & $=$ & $0,0.1 \cdot 1$ & $\left.0,7^{\prime}\right) 5+$ & Barcelona & $==$ & $0,0,25+$ & $0.534+$ \\
\hline celona & $=$ & $1,000 \cdot h$ & 1,000 & Guipúzcoa & $=$ & ים- רים & $0,5.7+$ \\
\hline & & & & & $=$ & $0.090+$ & $0,887+$ \\
\hline & & & & & $=$ & I. & I. \\
\hline
\end{tabular}

(1) Las provinrlas vienen sumadas unas a ntras. arumulánduse el total ie ludas lag

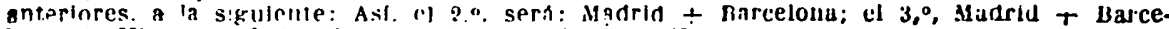
lona + vizcaya: el $4.0^{\circ}$, las anteriures, más Teuerife, etc: 
Contribución relativa ascendente por habitante en las provincias españolas y su población relativa y acumulación de las mismas. - Año 1941.

\begin{tabular}{|c|c|c|c|c|c|c|c|}
\hline \multirow{2}{*}{\multicolumn{2}{|c|}{ PROVINCIAS }} & \multicolumn{2}{|c|}{ Patentes } & \multirow{2}{*}{\multicolumn{2}{|c|}{ PROVINCIASS }} & \multicolumn{2}{|c|}{ Total las 5 rúbricas } \\
\hline & & $\begin{array}{l}\text { ranto por } \\
1 \text { de ia } \\
\text { población }\end{array}$ & $\begin{array}{l}\text { Tanto por } \\
1 \text { de la } \\
\text { contribucion }\end{array}$ & & & $\begin{array}{c}\text { Tanto por } \\
1 \text { de la } \\
\text { poblacion }\end{array}$ & $\begin{array}{c}\text { Tanto por } \\
1 \text { de la } \\
\text { contribucic }\end{array}$ \\
\hline Ciudad Real & $=$ & $0,02 I+$ & $0,046+$ & Lugo & $=$ & 0,020 f- & $0,007 \div$ \\
\hline Cuenca & $=:$ & $0,0.34$ & $0,011+$ & Orense & $=$ & $0,0.39+$ & $0,014+$ \\
\hline Teruel & $=$ & 0,043 & $0,0 I_{4}+$ & Almería & $=$ & 0,052 & $0,020+$ \\
\hline Toledo & $:=$ & 0,062 & $0,021+$ & Jaén & $=$ & $0,0 \Omega_{2} \div$ & $0,033+$ \\
\hline Zamora & $=$ & 0,074 & $0,025+$ & Granada & $=$ & $0, \mathrm{III}+$ & $0,046+$ \\
\hline Almería & $\therefore=$ & 0,088 & $0,031+$ & Albacete & $=$ & $0,125 \cdot T$ & $0,053 \dot{T}$ \\
\hline Badajoz & $=$ & $0,1: 7$ & $0,044+$ & Tenerife (S. C.) & $==$ & $0,140 \div$ & $0,059 \div$ \\
\hline Albacete & $\ddot{z}$ & 0,132 & $0,051+$ & Ciudad Real & $=$ & $0,161+$ & $0,069+$ \\
\hline Avila & $=$ & $0,14 \mathrm{I}-$ & $0,055+$ & Cuenca & $=$ & $0,174+$ & $0,075+$ \\
\hline lalajara & $\because=$ & $0,149-$ & $0,0.59+$ & Toledo & $:=$ & $0,193 \div$ & $0,084+$ \\
\hline Orense & $=$ & $0,167+$ & $0,068+$ & Córdoba & $=$ & $0,223+$ & $0,090 \div$ \\
\hline Cáceres & $=$ & 0,187 & $0,079+$ & Avila & $=$ & $0,2.32+$ & 0,104 \\
\hline $\mathrm{Cc}$ & $=$ & 0,222 & $0,097+$ & Murcia & $\because=$ & $0,260+$ & 0,118 \\
\hline Granada & $=$ & $0,251-$ & $0,153+$ & $\mathrm{Co}$ & $=$ & 0,295 & $0,136+$ \\
\hline León & $=$ & 0,270 & $0,123+$ & Teruel & $==$ & $0,304 \div$ & $0,141+$ \\
\hline Jaén & $=$ & 0,300 & $0,139+$ & León & $=$ & $0,323 \div$ & $0,151+$ \\
\hline Palencia & $=$ & $0,309-$ & $0,144+$ & tevedra & $:=$ & $0,348+$ & $0,165+$ \\
\hline Salamanca & $=$ & 0,324 & $0,154+$ & Cá & $=$ & $0,368+$ & $0,176 \div$ \\
\hline Huelva & $=$ & $0,33^{\circ}$ & $0,163+$ & Badajoz & $=$ & $0,397+$ & $0,192+$ \\
\hline M & $=$ & 0,365 & $0,181+$ & So & $=:$ & $0,403+$ & $0.196+$ \\
\hline Córdoba & $=$ & 0,395 & $0,202+$ & $\mathrm{Pal}$ & $=$ & $0,416+$ & $0,20,3+$ \\
\hline Murcia & $=$ & 0,423 & $0,222+$ & Huelva & $=$ & $0,430 \div$ & 0,2 ए 2 \\
\hline 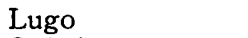 & $==$ & 0,413 & $0,2,37+$ & $\mathrm{Zat}$ & $=$ & $0,442+$ & $0,219+$ \\
\hline Ovi & $=$ & $0,476+$ & $0,263+$ & Al & $==$ & $0,466+$ & $0,2,33$ \\
\hline ider & $=$ & 0,492 & $0,275+$ & $\mathrm{Bu}$ & $z=$ & $0,481+$ & $0,242 \div$ \\
\hline dolid & $=$ & 0.505 & $0,286+$ & Lérida & $\therefore=$ & $0,493+$ & $0,249 t$ \\
\hline vedra & $=$ & 0,530 & $0,307+4$ & ga- & $=$ & $0,520+$ & $0,267+$ \\
\hline Hu & $=$ & $0,5.39 t$ & $0,314+$ & lajara & $:=$ & $0,528+$ & $0,273+$ \\
\hline S & $:=$ & $0,5.74-$ & $0,326+$ & $\mathrm{Ov}$ & $=$ & $0,56 \mathrm{I}+$ & $0,296+$ \\
\hline & $=$ & $0,5(0)-$ & $0,331+$ & $\mathrm{Ca}$ & $=::$ & $0,573+$ & $0,305 \div$ \\
\hline lón & $=$ & $0,572-$ & $0,342+$ & $\mathrm{H}_{1}$ & $\because=$ & $0,582+t$ & $0,312+$ \\
\hline lencia & $=$ & $0,622+$ & $0,380+$ & $\mathrm{nca}$ & $==$ & $0,597+$ & $0,324+$ \\
\hline govia & $=$ & $0,0,30+$ & $0,393+$ & Bale & $=$ & $0,613+$ & $0,3,36 \cdot L$ \\
\hline Tenerife (S. C.) & $=$ & $0,0.44+$ & $0,406+$ & $\mathrm{Ge}$ & $:=$ & $0,626+$ & 0,3160 \\
\hline Logroño & $=$ & $0.653-1$ & $0,415+$ & uder & $=$ & $0,042 \perp$ & $0,359 \div$ \\
\hline gona & $=$ & $0,666+$ & $0,428+$ & rona & $=$ & $0,655+$ & $0,369 \div$ \\
\hline te & $=$ & $0,690+$ & $0,452+$ & & $=$ & $0,663+$ & $0,375+$ \\
\hline & $\therefore$ & $0,702+$ & $0,464+$ & ncia & $==$ & $0,672+$ & 0,382 - \\
\hline $\mathbf{z}$ & $=$ & $0,726+$ & $0,48 z+$ & Cádiz & $:=$ & 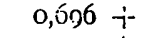 & 0,402 \\
\hline eares & $=:$ & $0,742+$ & $0,506+$ & & $=$ & $0,705+$ & $0,410 \div$ \\
\hline Palmas & $=$ & $0,755+$ & $0,523+$ & olid & $:=$ & $0,713+$ & $0,422+$ \\
\hline evilla & $==$ & $0,793+$ & $0,575 \cdot \vdash$ & ncia & $=-$ & $0,7 f 88+$ & $0,468+$ \\
\hline & $=$ & 0,813 & $0,6 \mathrm{xl}+$ & Sev & $=:$ & $0,806+$ & $0,505+t$ \\
\hline & $==$ & $0, \delta 25$ - & $0,6.35+$ & & $=-$ & $0.829+$ & $0,533+$ \\
\hline oza & $=$ & $0,849+$ & $0,665+$ & ona & $=$ & $0,905 \div$ & $0,703+$ \\
\hline & $=$ & 0,9 I I - & $0,7009+$ & úzcoa & $=$ & $0,318 \div$ & $0.734+$ \\
\hline ona & $=$ & $0,987+$ & $0,957+$ & & $=$ & $0.980+$ & $0,933+$ \\
\hline $\operatorname{coa}$ & $=$ & $I, \infty O$ & 1,000 & aya & $:=$ & $000^{\prime} 1$ & 1,000 \\
\hline
\end{tabular}

(1) I.as provincias vienen sumadas vnas a otras, acumulándose el total de todas las anteriores, a la siguiente: Así, el 2.0, será: Madrid + Barcelona: el $3:^{\circ}$, Madrid + Barcelona 
El primer cuadro estadístico contiene el tanto por uno de la población española correspondiente a cada provincia, con la riqueza imponible, rústica y urbana, total y por unidad provincia; la contribución total por unidad provincia y por habitante en cada una de ellas, rústica, urbana, industrial, utilidades y patentes. Ello permite ordenar las provincias españolas, en cada uno de los epígrafes, en orden ascendente, para darnos una primera idea de la estructura española de tales tributos, según las provincias, teniendo en cuenta su número de habitantes, y, en el caso de la riqueza imponible, rústica y su contribución, por kilómetro cuadrado, en cuyo último aspecto Albacete (307), Soria (361) y Teruel (377) son las consideradas como más pobres; y Sevilla (1.303), Valencia (1.982) y Pontevedra (1844) como las más ricas. En cambio, consideradas teniendo en cuenta el número de habitantes, las provincias estimadas como las más pobres en riqueza rústica son: Madrid $(3,94)$, Barcelona $(4,83)$ y Vizcaya $(5,16)$, y las más ricas: Palencia $(32,36)$, Guadalajara $(30,22)$ y Huesca $(29,81)$, ocupando Valencia $(17,32)$ un lugar casi central, contra lo que vulgarmente suele creerse.

$\mathrm{Si}$ atendemos a la riqueza urbana por habitante, ocupan los primeros lugares Madrid $(36,72)$, Barcelona $(29,50)$ y Guipúzcoa $(25,65)$, y los últimos, Orense $(1,95)$, Lugo $(2,28)$ y Pontevedra $(3,96)$.

Respecto a la contribución industrial por habitante, ocupan los primeros lugares las provincias de Barcelona (41.93), Guipúzcoa $(33,25)$ y Madrid $(32,69)$ (ocupando Vizcaya el cuarto lugar $(31,42)$, a pesar de lo que vulgarmente se cree), y son Almería $(6,12)$, Orense $(6,16)$ y Cuenca $(6,27)$ las que tienen los últimos lugares.

En la contribución por utilidades por habitante son Vizcaya $(197,01)$, Madrid $(174,33)$ y Guipúzcoa $(114,20)$ las tres primeras (ocupando Barcelona el cuarto lugar con 94,80), mientras que los últimos lugares corresponden a Orense. $(2,54)$, Navarra $(3,58)$ y Lugo $(4,16)$.

En la contribución por patentes son Guipúzcoa $(5,91)$, Barcelona $(5,18)$ y Madrid (5) las que ocupan los primeros lugares por habitante, y Ciudad Real $(0,62)$, Cuenca $(0,78)$ y Teruel $(0,82)$ las que ocupan los últimos.

Finalmente, considerando la contribución total de las cinco dichas por habitante, son Vizcaya $(263,23)$, Madrid $(252,67)$ y Guipúzcoa $(\mathbf{1 8 5 , 5 1 )}$ las tres primeras (Barcelona ocupa el cuarto lugar con 176,24 , contra lo que vulgarmente suele creerse), y Lugo $(28,03)$, Orense $(28,49)$ y Almería $(32,37)$ las tres últimas. 
Estos datos nos dicen muy claramente la gran diferencia que existe en las provincias de España entre los intervalos o campos de variación de la tributación por habitante, en las diversas contribuciones consideradas; pues mientras la rústica oscila entre 3,94 para Madrid y 32,36 para Palencia, o sea de uno a ocho, aproximadamente, la urbana varía de 1,95 en Orense a 36,72 en Madrid; o sea de uno a diez y ocho, próximamente; la industrial lo hace de 6,12 en Almeria a 41,93 en Barcelona, o sea de uno a siete, aproximadamente; la de utilidades se mueve entre 2,54 en Orense y 197,01 en Vizcaya, o sea de uno a setenta y siete; la de patentes lo hace de 0,62 en Ciudad Real a 5,91 en Guipúzcoa, o sea, más de uno a nueve, mientras que la contribución total se mueve entre el mínimo de 28,03 en Lugo y el máximo de 263,23 en Vizcaya, o sea de uno a nueve, aproximadamente.

Según estos datos, parece a primera vista que la concentración de la contribución por provincias y habitante, sigue, de menor a mayor, el siguiente orden:

\author{
Industrial, \\ Rústica, \\ Total, \\ Patentes, \\ Urbana, \\ Utilidades ;
}

pero hay que tener mucho cuidado con una tal afirmación, porque puede conducir a errores graves, como de hecho conduciría en el caso de España, conforme vamos a demostrar en este estudio.

El cuadro II estadístico, contiene, como claramente se indica en una nota, para cada una de las contribuciones, dos series estadísticas elaboradas con los datos del cuadro I. El primer dato de la primera de estas series es el tanto por uno de la pob!ación que respecto a la total de todas las provincias de España, consideradas en el epígrafe respectivo, tiene la provincia que ocupe el ínfimo lugar en la serie del cuadro I "Contribución por habitante"; y el primer dato de la segunda serie es el correlativo en la columna "tanto por uno de la contribución" en el cuadro I. El segundo dato de la primera serie es la suma del primero y del que ocupa el lugar segundo, aplicando el mismo criterio; y el segunda dato de la segunda serie es la 
suma de los correlativos o correspondientes a los de la primera. El tercero, cuarto, etc., se obtienen mediante las sumas acumu'adas de los tres, cuatro, etc., respectivamente, que ocupan los lugares ascendentes, aplicando el criterio dicho. No hemos añadido otro cuadro con dichas ordenaciones ascendentes, de las cuales por suma acumulada, se obtiene el II, para no hacer demasiado largo nuestro trabajo para el lector.

Como consecuencia de la manera como se han obtenido los datos de las dos series del cuadro II, para cada clase de contribución, resulta evidente que cada par de datos correspondientes en dichas series (para cada clase de contribución), puede considerarse como coordenadas, cartesianas rectangulares, tomando como eje de abscisas el tantn por uno de la población y, como de ordenadas, el tanto por uno de la contribución correspondiente; con lo cual resultará un diagrama cuyo primer punto representa el tanto por uno de la contribución total (en cada clase de contribución) asignado a la provincia que paga menos contribución por habitante, correspondiente al tanto por uno que respecto a la población global de todas las provincias consideradas, tiene la provincia que ocupa el referido ínfimo lugar. El segundo punto del diagrama (siempre en cada clase de contribución) es el tanto por uno de la contribución total que pagan entre las dos provincias que satisfacen menos contribución por habitante, correspondiente al tanto por uno que respecto a la población global de todas las provincias consideradas tienen entre las dos provincias que ocupan los dos lugares ínfimos. $Y$ así sucesivamente, el punto tercero, cuarto, etc., en cada clase de contribución.

Con este procedimiento están construídos los diagramas del gráfico adjunto 




en el cual se supone, en atención al número de datos, que se trata de una línea prácticamente continua, aunque esto es sólo aproximadamente.

Esto supuesto, y recordando lo que es y lo que significa el concepto de CONCENTRACION, (cuya técnica está desarrollada en nuestro "Tratado de Estadística Matemática") en Economía y Estadística, resulta que las líneas de nuestro diagrama son líneas de concentración de la contribución de las diversas clases de tributos considerados, las cuales-en cuanto la contribución es índice de posesión de riqueza-, representan la concentración de riqueza correspondiente a las provincias de España, tomando como elemento unidad la provincia.

Por consiguiente, aplicando la consabida técnica a tales líneas, resulta que, aproximadamente, el índice de concentración de cada una de las contribuciones consideradas en este estudio es el área de 710 la superficie comprendida entre la recta OP y la línea correspon- 
diente a cada clase de contribución, dividida por el área del triángulo $O P Q=1 / 2$, por ser $O Q=Q P=1$.

Tenemos, pues, los siguientes resultados, aproximadamente.

Clase de contribución

Rústica ............

Urbana . . . ..........

Industrial . . . . . . . .

Patentes .............

Utilidades . . . ........

Total ..............

\section{Subindice de concentración}

0,27

0,41

0,34

0,31

0,61

\section{0,37}

De este Cuadro se infiere el verdadero orden de la concentración de las contribuciones consideradas, el cual no es el que hubiera podido parecer al observador superficial, desconocedor de la técnica estadística.

Ello nos dice que, en cuanto la contribución es signo de riqueza en las provincias españolas, es en la riqueza rústica en donde existe la menor concentración y que la máxima corresponde a la que se refiere al epígrafe "Utilidades", siendo mucho mayor la de Urbana que la de Industrial y casi el doble que la de Rústica.

Advertimos de nuevo al lector que nuestro estudio sólo se refiere a la provincia como elemento estadístico; y por tanto que nuestros resultados no pueden extenderse, sin el previo estudio correspondiente, a la concentración de riqueza de un país en el sentido de Wifredo Pareto. Tal estudio es técnicamente más sencillo que el nuestro, y para hacerlo se precisa conocer el número de contribuyentes y la cuantía de la contribución de cada uno, en cada epígrafe, para con tales datos obtener el tanto por uno que de cada clase de contribución total pagan entre todos los contribuyentes que paguen contribución no superior a $X$ (variando $X$ desde la mínima a la maxima) y el tanto por uno que tal número de contribuyentes representa respecto al número total de todos ellos, en cada clase de contribución. En la práctica se toma un número de valores, $\mathrm{X}$, suficiente para que el diagrama resulte prácticamente una línea continua. Ordenadas ambas series en orden creciente, y obtenidas, mediante la acumulación arriba expuesta, las dos series de coordenadas (tanto por uno de la población contribuyente, en cada clase de contribución, con cuota $0<x<1$, y tanto por uno que de la contribución global 
le corresponde), se construye el gráfico y se calculan los índices de concentración exactamente igual que en nuestro caso estudiado. ¿Cuándo tendremos los datos necesarios para calcular los índices de concentración dichos y mediante el'os los de la riqueza española correspondiente?

\section{RES U MEN}

A falta de los datos necesarios para calcular los índices de concentración de las diversas clases de contribución (y con ellos los de la concentración de la riqueza correspondiente en el sentido de Wifredo Pareto, en cuanto la contribución es índice de riqueza en España) relativos a nuestro país, hemos hecho el estudio tomando como elemento unidad la provincia española, con los datos mejores y más completos que hemos podido haber; y hemos obtenido el siguiente cuadro de índices aproximados:

\begin{tabular}{|c|c|}
\hline Clase de contribución & Subíndice de concentración \\
\hline Rústica & 0,27 \\
\hline Urbana. & 0,41 \\
\hline Industrial . . . . . & 0,34 \\
\hline 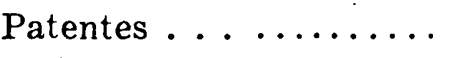 & 0,31 \\
\hline Utïidades . . ....... & 0,61 \\
\hline Total . & 0,37 \\
\hline
\end{tabular}

advirtiendo que el índice cero significa la equidistribución, y el índice uno, la concentración máxima que correspondería al caso en que to 'a la contribución fuera pagada por una so'a provincia española, en cada clase de contribución.

Estimamos evidente una gran disparidad entre la opinión generalizada en España sobre esta materia y los resultados indiscutibles que hemos obtenido.

\section{CUESTIONES PROPUESTAS}

De nuestro estudio se infieren casi espontáneamente, entre otros, los siguientes problemas a reso'ver:

1. ¿ ¿Son nuestras clases de contribución índices adecuadus do 712 la riqueza correspondiente a tales tributos? 
2. ¿Es económicamente buena la estructura de nuestros índices de contribución tributaria?

3. ¿ ¿Existe sistema económicofinanciero racional entre las diversas clases de nuestra tributación, en orden a la consecución de una distribución de riqueza, encaminada al logro del progreso económico social estable?

Olegario fernández Baños. 